A N N A L E S Annales de Bretagne et des Pays de l'Ouest

Anjou. Maine. Poitou-Charente. Touraine

123-3 | 2016

La Grande Guerre, inspiration des artistes de 1914 à

nos jours

\title{
Mémoires du camouflage de la Grande Guerre dans l'art contemporain
}

The Impact of Great War Camouflage on Contemporary Art

\section{Renaud Bouchet}

\section{(2) OpenEdition}

Journals

Édition électronique

URL : http://journals.openedition.org/abpo/3412

DOI : $10.4000 / a b p o .3412$

ISBN : 978-2-7535-5229-6

ISSN : 2108-6443

Éditeur

Presses universitaires de Rennes

Édition imprimée

Date de publication : 31 octobre 2016

Pagination : 167-185

ISBN : 978-2-7535-5220-3

ISSN : $0399-0826$

Référence électronique

Renaud Bouchet, « Mémoires du camouflage de la Grande Guerre dans l'art contemporain », Annales de Bretagne et des Pays de l'Ouest [En ligne], 123-3 | 2016, mis en ligne le 31 octobre 2018, consulté le 19 avril 2019. URL : http://journals.openedition.org/abpo/3412; DOI : 10.4000/abpo.3412 


\title{
Mémoires du camouflage de la Grande Guerre dans l'art contemporain
}

\author{
Renaud BouchET \\ Maître de conférences en histoire de l'art contemporain, CERHIO UMR 6258, \\ université du Maine (Le Mans)
}

Le camouflage matériel appliqué durant la Première Guerre mondiale est apparu comme une nécessité immédiate, tout particulièrement dans le contexte d'un conflit de position révélant la vulnérabilité des forces exposées. Fait inédit dans l'histoire militaire, sa théorisation et son application ont été confiées à un ensemble d'artistes et artisans d'art dont les compétences spécifiques ont été sollicitées notamment pour la dissimulation d'objectifs soit par fusion homotypique avec le milieu d'implantation, soit par destructuration des formes révélatrices au moyen de procédés graphiques et chromatiques.

Les plasticiens de la seconde moitié du Xx $x^{\mathrm{e}}$ siècle ont pu rencontrer ce champ du camouflage d'abord développé pour la guerre terrestre dans l'optique défensive et offensive. De 1961 à 1964, le français Alain Jacquet l'a ainsi assumé dans le contexte de création artistique, en obtenant avec ses Camouflages des surfaces traitées selon un principe de superposition et juxtaposition de formes colorées appliqué à des œuvres représentatives voire iconiques de la modernité picturale ou encore du Pop Art exactement contemporain, telles Luxe, Calme et Volupté d'Henri Matisse et Little Aloha de Roy Lichtenstein (1963 et 1964). À la même époque, il conçoit le projet, jamais concrétisé, d'un recouvrement des murs de la galerie parisienne Breteau avec des couleurs et motifs de camouflage pour y accrocher des tableaux constitués de toile de camouflage montée sur châssis. Pierre Buraglio, de 1966 à 1968, suit une démarche comparable avec ses " exercices de camouflage " parodiant la peinture géométrique néoplasticienne de Piet Mondrian. Dans les années 1980, Andy Warhol, qui a compté avec Roy Lichtenstein parmi les visiteurs de l'exposition des Camouflages de Jacquet organisée à la Galerie Iolas de New York en mai $1964^{1}$, investit lui aussi le

1. Alain Jacquet accueillit le public présent au vernissage de son exposition vêtu d'un costume camouflé, confectionné à partir de toile de parachute. 
motif camouflé, employé selon un procédé autonome de superposition et de recouvrement, notamment avec Self-portrait et Camouflage (1986).

Il ne semble pas toutefois que ces artistes, comme encore plus récemment Éric Rutten avec son Camouflage primaire Espagne désert (2004), Karine Meyffret avec sa Mini-tente de camouflage (c. 2012), Sylvain Lang avec son Arbre camouflé ou Mondrian camouflé (2000), Jean-Pierre Raynaud avec son Pot camouflage (2008) et encore Tony Garifalakis avec son voile d'observation The Hills Have Eyes - Woodland (2012), aient eu pleinement conscience de ce qu'ils devaient aux camoufleurs de la Grande Guerre et en particulier aux camoufleurs français, initiateurs et chefs de file de la dissimulation militaire moderne en contexte terrestre ${ }^{2}$. Pour Jacquet par exemple, les Camouflages du début des années 1960 font certes écho à un contexte guerrier, mais le plus immédiat. "Le problème pour moi, à cette époque, était réellement le camouflage militaire. J'ai commencé ces travaux alors qu'on était à la fin de la guerre d'Algérie ", rapporte-t-il. Un référentiel contextuel qui n'est cependant pas exclusif, ainsi qu'il l'indique à Catherine Millet : " J'aimais cette idée du camouflage qui me permettait par exemple de reprendre, immédiatement après leur création des tableaux du pop art. [...] Je pouvais introduire une notion critique au sujet d'un art qui lui-même se présentait comme critique par rapport à la société de consommation ${ }^{3}$. " D'autres artistes ont en revanche clairement corrélé leurs pratiques empruntant au camouflage militaire et à la contribution des premiers artistes camoufleurs, ainsi qu'en témoignent les quelques cas qui vont être évoqués dans la présente étude.

\section{Postérités postmodernes du camouflage terrestre}

Dès le début de la Grande Guerre, des soldats, des pièces d'artillerie et leurs caissons à munition sont cachés derrière des feuillages et des bottes de paille ou de foin ${ }^{4}$, permettant d'annuler ou de limiter la capacité de l'adversaire à détecter depuis le sol la forme et l'ombre portée des hommes et objets dissimulés. Ces pratiques sont le fait de combattants non formés agissant spontanément, ou bien de soldats ayant reçu une instruction spécifique éprouvée notamment dans le contexte des grandes manœuvres du début du $\mathrm{xx}^{\mathrm{e}}$ siècle. Mais peu crédible hors des lieux à vocation agricole et hors contexte saisonnier, peu apte à tromper une observation aérienne

2. Le sculpteur-camoufleur Henri Bouchard, spécialiste des faux arbres-observatoire, écrit en à la fin de 1915 : " Nous faisons ce que les Allemands n'ont pas encore trouvé, ils sont peut-être plus scientifiques, mais nous sommes plus ingénieux. " (Henri Bouchard, lettre d'octobre 1915, Archives Henry Bouchard, Paris.)

3. JACQUET Alain, dans Millet, Catherine, "Alain Jacquet. Voyage au centre de la terre", ArtPress, Paris, no 146, avril 1990, p. 16.

4. Voir notamment les photographies de pièces d'artillerie camouflées publiées dans les hebdomadaires L'Illustration du 7 novembre 1914 ( ${ }^{\circ}$ 3776) et Sur le Vif du 16 janvier 1915 ( ${ }^{\circ}$ 10), ainsi que LINTIER, Paul Michel, Avec une batterie de 75, ma pièce : Souvenirs

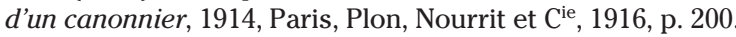


de plus en plus performante, cette option du paravent végétal ne pouvait constituer qu'un recours ponctuel. Celle d'un camouflage artificiel a donc été envisagée et mise en pratique de façon presque contemporaine aux premières actions de dissimulation au moyen d'éléments naturels prélevés dans l'environnement immédiat. Trois hommes sont généralement tenus pour en être les inventeurs simultanés, à commencer par le peintre, dessinateur et illustrateur Lucien-Victor Guirand de Scévola, figure de la vie artistique et mondaine parisienne de l'avant-guerre ${ }^{5}$.

Au commencement du conflit, le maréchal des logis du Génie Guirand de Scévola assure la liaison téléphonique entre l'état-major de Pont-àMousson et une pièce d'artillerie de 155 long. Constatant la vulnérabilité de la position repérée par l'aviation adverse après chaque tir, il imagine de la dissimuler sous des étoffes bariolées dont les teintes se confondent avec celles du terrain, et dont les motifs modifient la perception visuelle de l'objet caché. Après avoir vérifié son efficacité, il étend le dispositif aux artilleurs eux-mêmes, qui se voient dotés de blouses camouflées cachant les mains et les visages.

En septembre 1914, en réaction à la mort de l'un de ses amis chef de pièce d'artillerie victime d'un bombardement d'aviation, le maréchal des logis au $6^{\mathrm{e}}$ régiment d'artillerie à pied Eugène Corbin, propriétaire et directeur des Magasins Réunis de Nancy, peintre et mécène des artistes de " l'École de Nancy, Alliance provinciale des industries d'art " fondamentalement inspirés par les formes animales et surtout végétales, conçoit lui aussi le camouflage des canons et des servants au moyen d'une toile peinte selon le principe de mimétisme homotypique. Il sollicite pour sa réalisation l'une de ses relations de longue date, le peintre et décorateur Louis Guingot.

Depuis le mois d'août, celui-ci mène de son côté des recherches croisées sur le phénomène mimétique chez le caméléon et sur la technique picturale du pointillisme qui subdivise la forme en une multitude de points de couleur. Combinant ses travaux à son invention de 1902 (un procédé de peinture à l'acide fluorydrique pour tout type de tissus, résistant à la lumière comme à un nettoyage à l'eau de javel), et s'inspirant peut-être du vêtement de chasse mis au point par le peintre Émile Friant ${ }^{6}$, il réalise une

5. Sur les origines controversées du camouflage militaire artificiel, voir PECCATTE, Patrick, "Cubisme et camouflage - un mythe de l'histoire de l'art ", Dejavu.hypothèse.org, 14 avril 2015, [http://dejavu.hypotheses.org/2220]. Consulté le 2 décembre 2015. Voir aussi Coutin, Cécile, "Invention du camouflage ", Tromper l'ennemi. L'invention du camouflage moderne en 1914-1918, Villers-sur-Mer/Paris, Pierre de Taillac/Ministère de la Défense, 2012, p. 21-31.

6. Au tout début du $\mathrm{xx}^{\mathrm{e}}$ siècle, Émile Friant mit au point une veste dont les rayures verticales permettaient une meilleure dissimulation du chasseur derrière des arbres. Mais cette invention resta très confidentielle et ne fut sans doute pas portée à la connaissance de l'armée. Voir ConTE, Albert, Louis Guingot (1864-1948) et les autres : peintre - inventeur du camouflage de guerre en 1914, A. Conte, 1996, p. 16. Cité dans THIERY, Frédéric, "La première veste de camouflage de guerre du monde est inventée par Louis Guingot ", Guerres mondiales et conflits contemporains, 3/2007, $\mathrm{n}^{\circ} 227$, p. 7-21, [www.cairn.info/revueguerres-mondiales-et-conflits-contemporains-2007-3-page-7.htm]. Consulté le 20 février 2011. 
veste camouflée associant trois couleurs : le vert pré, le brun-rouge et un bleu sombre appelé "bleu Guingot ", employé par l'artiste dans ses décors de théâtre notamment pour souligner les ombres. Corbin, qui a fourni la veste confectionnée dans son atelier de couture des Magasins Réunis, la fait aussitôt parvenir aux Services des Armées à Paris qui, après le prélèvement d'un échantillon de 14 x 15,5 cm, la restituent à Guingot tout en l'informant qu'il ne fera pas fait usage de son invention ${ }^{7}$.

Toujours en septembre 1914, Guingot et Corbin - à moins qu'il ne s'agisse de Guirand de Scévola conformément au récit livré par ce dernier en $1950^{8}$ - obtiennent du colonel Fetter, commandant du $6^{\mathrm{e}}$ régiment d'artillerie lourde, l'autorisation de procéder à un premier essai de dissimulation grandeur-nature d'un canon et de ses servants au fort de Domgermain, à Toul. Pour cet essai, une grande toile confectionnée aux Magasins Réunis est tendue au-dessus d'un canon peint aux couleurs dominantes de l'environnement, et 5 de ses 10 servants sont dotés d'uniformes assortis. Survolant la zone en avion à seulement $300 \mathrm{~m}$ d'altitude, le fils du colonel Fetter ne peut remarquer que les artilleurs revêtus de l'uniforme réglementaire. Devant l'efficacité du dispositif le colonel Fetter charge un groupe d'ouvriers de l'arsenal de Toul de peindre des toiles de camouflage sous la direction de Corbin et Guingot. Quatre artistes dont Guirand de Scévola se joignent à cette "Équipe d'art du 6e Régiment d'Artillerie à Pied ", qui devient rapidement "L'Équipe de Camouflage ", une dénomination accueillie " avec quelque ironie de la part des officiers de carrière ${ }^{9}$ ".

Près d'un siècle après ces évènements structurant la préhistoire du premier service de camouflage de la Grande Guerre, du 5 au 27 février 2011, s'est tenue à La Douëra de Malzéville, près de Nancy, l'exposition de groupe Voyage en Camouflistan, initiée par l'artiste nancéen Gérard Didierjean alias Lakonik. Le texte de présentation produit par ce dernier établit, sur le ton humoristique, un lien explicite entre le camouflage de la Première Guerre mondiale et les contributions des 23 plasticiens, photographes et designers participant à l'exposition parmi lesquels Olivier Bello, Stéphane Lempereur, Éric Didym, Christophe Gile ou encore Louise Pressager, auteure pour l'occasion d'une Vache de guerre en tissu, découpée dans une chemise camouflée qui sera portée par Lakonik à au moins une occasion ${ }^{10}$ (figure 55). Celui

7. Voir THIERY, Frédéric, "La première veste... ", op. cit.

8. Voir GuiRAnd de SCÉVOLA, Lucien Victor, Souvenirs du camouflage (1914-1918), Revue des Deux Mondes, Paris, janvier 1950, p. 717-733. On opposera à ce témoignage celui livré par Henri Guingot dans "Les artistes et la guerre : le camouflage ", [discours], Bulletin Trimestriel de la Société d'Émulation du département des Vosges, Épinal, janvier 1934, p. 17. Henri Guingot est le fils de Louis Guingot. Ce statuaire formé à l'École des Beaux-Arts de Nancy a été associé aux travaux de son père menés au sein de la Section de camouflage de l'armée française.

9. GUINGOT, Henri, "La première veste... ", op. cit., p. 17.

10. Si l'on en croit l'artiste, il faut y voir ni une référence au découpage du prototype de veste camouflée conçu par Louis Guingot, ni une influence des vaches-leurres réalisées par la Section de camouflage : " [...] ma Vache de guerre était une petite installation totalement dissociée de la thématique de la Première Guerre Mondiale. [...] il fallait que 
qui se présente alors comme « l'Ambassadeur officiel de la République du Camouflistan ", future entité territoriale et culturelle fictive, écrit notamment :

« En 1915, le peintre Louis GUINGOT en essuyant ses pinceaux sur sa blouse s'est fondu dans le paysage qu'il peignait. De ce fait, il a imaginé que ce motif qui rend l'homme invisible pouvait sauver des milliers de vies humaines. Or, à ce moment, notre pays envoyait sur le front ses hommes infortunés vêtus des couleurs nationales; le rouge garance et le bleu pétrole (déjà). L'état-major, sûr de son intelligence, a bien sûr refusé l'idée saugrenue de déguiser ses combattants en arbres feuillus. Il était alors plus noble de mourir en héros au combat. [...] Cependant, le fameux lieutenant JeanBaptiste Eugène CORBIN, mécène de l'école [de Nancy] et capitaine de grande surface, a testé sur les toiles qu'il vendait ce motif abstrait pour en connaître les vertus. Son fils, alors pilote, en visualisant le sol à trois cents mètres d'altitude n'a remarqué que les deux soldats (sur cinq) qui n'étaient pas "camouflés". Cette découverte a été le déclencheur, malgré la fin de la guerre qui s'annonçait, de l'acceptation de cette icône que l'on appelle aujourd'hui encore le "camouflage". [...]. À la suite de ces expériences vestimentaires destinées aux tueurs ou à leurs victimes, quatre ateliers de camouflages ont été créés en Camouflistan. Beaucoup de Camouflistanais et Camouflistanaises y ont travaillé à l'instigation de prestigieux artistes de l'époque; Fernand Léger, Georges Braque, André Mare, Marcoussis, Raymond Duchamp-Villon (le frère de Marcel), Joseph Porphyre Pinchon (le créateur de Bécassine et de Frimousset) pour les plus connus, puis des courants artistiques; le cubisme, l'expressionnisme allemand, le futurisme italien et le vorticisme anglais. Tout ce beau monde camouflait dans la bonne humeur avec parfois des désaccords esthétiques qui différenciaient leurs écoles. Ils inventaient sans le savoir le land $\operatorname{art}^{11}$."

Combinant des teintes beiges, noires et vertes, la propre production camouflée de Lakonik, présentée plus largement au sein d'une installation monumentale à la Demeure du Chaos, près de Lyon, en 2011, reste fidèle à l'objectif initial de dissimulation propre au camouflage militaire. Elle investit en outre les mêmes supports, à savoir l'objet lui-même (avion, char, container...), la toile et le filet, pour créer un environnement répondant aux bivalences ironiques du territoire et de la mentalité camouflistanaises :

" Aujourd'hui en 2050, la République du Camouflistan, après avoir traversé quelques crises économiques, sociales, identitaires, guerrières, ministérielles, est devenue un territoire où il fait bon vivre. Il y a certes des

je produise quelque chose sur le thème du camouflage, et comme je réfléchis toujours par analogie et que j'ai un fâcheux penchant pour les jeux de mots, j'ai pensé aux taches des vaches vosgiennes. C'est totalement par hasard que j'ai découpé ma vache dans une veste militaire, c'est ce que j'ai trouvé de moins cher dans un surplus. Et c'est également fortuitement que j'ai fait cadeau à l'organisateur de l'expo de la veste en question dont j'avais rapiécé le "trou" avec du tissu rouge sang. Par la suite j'ai pu assister à une conférence sur Louis Guingot, je crois qu'elle était donnée par Marcel Cordier, un spécialiste local [...]. " (Louise Pressager, propos recueillis par l'auteur, 4 décembre 2015.)

11. LAKONIK, "Voyage en Camouflistan ", préface de l'exposition éponyme, La Douëra, Malzéville, 5-27 février 2011. Reprod. dans [http://www.verdun-meuse.fr/index.php?qs=fr] grande-guerre/exposition-voyage-en-camouflistan]. Consulté le 27 février 2011. 
contraintes et des dangers pour ceux qui veulent s'y rendre mais il y a aussi beaucoup de satisfactions touristiques : ses paysages, son infrastructure, ses commerces, ses produits de consommation, ses artistes, sa prostitution... Les Camouflistanais sont des gens rustres en raison de la situation géographique rude de leur pays. Mais à l'instar de leur Président, ils sauront vous accueillir. Sans excès de générosité : ils sont avant tout des hommes et des femmes virils. L'homme moderne qui les dirige et les protège a fait de cette République une véritable entreprise qui exporte ses produits partout dans le monde. Cette exposition montre quelques aspects touristiques et commerciaux qui pourraient, vous donner à vous aussi l'envie d'y passer un agréable séjour lors de vos prochaines vacances familiales... Alors, parez vous de vos plus beaux effets camouflants et venez découvrir ce magnifique pays : LE CAMOUFLISTAN ${ }^{12}$."

En mars 2011, Jean Claude Le Gouic fait également usage de filets de camouflage présentés en autonomie avec ses Camouflages fluorescents (figure. 56) installés sur le site de la friche postindustrielle des Perles, à Barjols (Var). Le texte de l'artiste aixois présentant l'intervention in situ établit là-aussi un lien immédiat entre les œuvres montrées et le camouflage du premier conflit mondial, en dépit du paradoxe que constitue, tout au moins en contexte terrestre, un " camouflage fluorescent ". Citons-en les lignes introductives : "Les artistes ont entretenu une relation suivie avec les pratiques de camouflage. L'armée française, durant la grande guerre, utilisa les compétences de différents artistes dont Fernand Léger, pour développer les motifs pour les tenues des soldats et les peintures sur le matériel militaire. " Comme celui du Voyage en Camouflistan, le texte confère ensuite une dimension artistique au camouflage de la Grande Guerre et à ses prolongements avant-gardistes qui le réactualisent :

"Les militaires se sont mis à penser en termes d'arts plastiques. Pour casser les formes, pour les rendre moins discernables, ils ont développé le principe FORMATT pour Forme, Ombre, Reflet, Mouvement, Arrière-plan, Tonalité, Traces. Très vite de leur côté les artistes vont se réapproprier et détourner les motifs militaires : ce fut le cas dans les années soixante de Roy Lichtenstein, Andy Warhol, Alain Jacquet, et aussi plus récemment de Michel Aubry, Thomas Hirschorn... Après celui de la chasse, le monde de la mode s'est également intéressé à ces motifs ${ }^{13}$."

Les deux textes de présentation cités ici ont certainement vocation à donner au public des clefs de compréhension utiles, en dépit des erreurs chronologiques et factuelles importantes qu'ils contiennent : Guingot, par exemple, a conçu son tissu camouflé dit "Léopard " dès la fin de 1914, et Léger n'a jamais intégré le Service du camouflage de l'armée française en dépit de ses demandes répétées. Mais l'énumération de figures du monde artistique associées à trois temporalités, celle de la Grande Guerre, celle

\section{Ibidem.}

13. LE GouIC, Jean-Claude, " Camouflages fluorescents. Installation. Lumière blanche, lumière noire ", Les Perles, Barjols, mars 2011, [http://jc.legouic.free.fr/barjolstxt.html]. Consulté le 24 mai 2011. 
des années 1960-1970 et enfin celle des années 2010, découle peut-être d'une autre intention, à savoir l'inscription des œuvres conçues et présentées par Lakonik et Le Gouic dans une véritable tradition artistique incontestable, propre à les légitimer. Et cette légitimation, intentionnellement recherchée ou non, est renforcée par un rappel territorial à deux échelles. S'il évoque le rôle de l'Expressionnisme allemand, du Futurisme italien et du Vorticisme anglais, le texte de Lakonik s'ouvre sur ces mots se rapportant à la République Démocratique et Populaire du Camouflistan de 2050, probable projection identitaire de la Lorraine : " Ce qui est aujourd'hui notre fierté nationale est né dans un village de la banlieue de Malzévile : Nancy ${ }^{14}$."

Le texte, à cet égard, ajoute un hommage nancéien supplémentaire à Eugène Corbin et surtout à Louis Guingot considéré, particulièrement à l'échelle régionale et en conformité notamment avec les conclusions de l'érudit René Nouveau ${ }^{15}$, comme le véritable inventeur du camouflage artificiel, création pour laquelle, rappelle Lakonik, il « n’a jamais touché la moindre royaltie ${ }^{16}$ ". Citons à titre d'exemple le texte de la plaque murale apposée sur la maison de l'artiste située au n ${ }^{\circ} 10$ de la rue d'Auxonne, dans la banlieue de Nancy : " Ici dans sa chaumière où il vécut de 1905 à 1923 Louis Guingot, artiste peintre de l'école de Nancy inventa le camouflage de guerre en 1914. Don de "Terre Lorraine" 17. " Une paternité étendue à Corbin par Henri Guingot dans son discours de 1934 déjà cité, lequel s'achève sur ces mots : " J'ai tenu à démontrer que c'est à deux Lorrains que revient l'honneur d'avoir imaginé et créé le principe du camouflage utilisé pendant la grande guerre, par les armées de toutes les nations belligérantes, et, qui plus est, l'un des deux, celui qui a peint la première toile couleur de terrain est Vosgien, Vosgien de pure race, fier de l'être ${ }^{18}$. "

Il est toutefois à signaler l'existence d'un précédent aux premières solutions de camouflage artificiel mises au point par Guirand de Scévola, Corbin et Guingot, constitué par le " filet-abri de camouflage " conçu dès 1912 par le commandant Anatole Kopenhague. Testé avec succès en octobre de la même année et de l'année suivante, le dispositif élaboré à partir de fragments de toile cirée vert foncé et de paille naturelle de couleur vertjaune est cependant écarté par le ministère de la Guerre le 22 août 1914 aux motifs d'un coût de fabrication jugé trop élevé pour un usage que l'on imagine alors trop restreint : la guerre nouvelle, de l'avis majoritaire, sera

14. LAKONIK, "Voyage... ", op. cit.

15. Voir Nouveau, René, "Le camouflage de guerre ", Études touloises, Toul, nº 4, fasc. 2, 1975 , p. $42-45$.

16. LAKONIK "Voyage... ", op. cit.

17. On notera en revanche que la plaque visible sur la maison natale de Guingot, au 90 rue Charles de Gaulle à Remiremont, ne l'associe pas aussi explicitement à la pratique du camouflage : «Ici naquit et vécut Louis Guingot (1864-1948) Artiste Peintre et Inventeur. Don de ses Amis."

18. Guingot, Henri, " les artistes et la guerre... ", op. cit., p. 17. 
en effet mobile et de courte durée ${ }^{19}$. En janvier 1915, les autorités militaires demanderont à Kopenhague de leur fournir la documentation constituée autour de son invention, sans pour autant lui confier un rôle de premier plan dans l'expérimentation, la production et l'application des procédés artificiels de dissimulation au sein du Service du camouflage.

Plus globalement, le texte de Le Gouic évoque implicitement et à juste titre le rôle pionnier des artistes camoufleurs nationaux, en rappelant que le terme "français "camouflage" n'ayant pas d'équivalent dans de nombreuses langues, fut adopté par les armées étrangères ${ }^{20}$ ». Signe de la prévalence de leur compétence initiale, les camoufleurs français dirigés par Guirand de Scévola ont aidé à la mise en place opérationnelle des services de camouflage terrestre dans les armées alliées, tel André Mare au sein de l'armée anglaise au cours de l'année 1916.

Cette primauté effective du camouflage français va toutefois disparaitre avec le conflit qui a permis son affirmation, faute de volonté réelle de maintenir au-delà de l'engagement le savoir-faire de ses concepteurs et praticiens, et d'adapter leurs solutions à de nouvelles nécessités imposées par les évolutions technologiques, en particulier dans le domaine de l'aviation : la protection des combattants, des axes de communications, du matériel, des transmissions et des populations civiles à bien plus grande échelle. Dans la période de l'entre-deux-guerres, la politique en faveur du camouflage se réduit ainsi à trois actions principales, à savoir la publication par le ministère de la Guerre d'un petit manuel d'instruction reprenant quelques généralités sur le camouflage du premier conflit (Instruction provisoire technique sur le camouflage, 1930), la dotation généralisée des troupes en uniformes kaki (vers 1935), et le camouflage par taches vert foncé, bleu foncé et chamois sur les appareils de la chasse (1938). Par la force des choses, le commandement français renouera avec le camouflage militaire dans la phase préparatoire du second conflit mondial mais l'incompétence avec laquelle il sera appliqué ne sera pas compensée par la réintégration de quelques artistes camoufleurs de 1915-1918, dont Guirand de Scévola et Corbin, alors âgés de 70 et de 72 ans. Nombreux pourtant ont été les observateurs militaires qui, dans les années 1920 et 1930, ont insisté sur la nécessité de faire du camouflage une arme constamment opérationnelle. Le commandant Lalance, par exemple, émettait cette préconisation dès 1923 :

" [Le] procédé de défilement doit être moins que jamais négligé, doit même être étudié et organisé pendant la paix. Le cadre des camoufleurs, formés surtout de réservistes, doit être tenu en haleine et préparé à une guerre

19. Une autre raison du rejet de la solution proposée par Anatole Kopenhague réside sans doute dans la répugnance à se dissimuler face à l'adversaire. Dans la tradition militaire, la visibilité des troupes est à la fois une marque de courage et donc d'honneur, et aussi une arme psychologique destinée à impressionner l'adversaire.

20. LE GouIC, Jean-Claude, "Camouflages fluorescents... ", op. cit. Le terme argotique " camouflage " a été employé à partir des années 1830, initialement dans le milieu théâtral, comme synonyme de "déguisement " ou "falsification". 
Mémoires du camouflage de la Grande Guerre dans l'art contemporain

où son rôle paraît devoir être bien plus important que dans la dernière, en raison de la forme aérienne qu'elle prendra certainement ${ }^{21}$. "

\section{Postérités du camouflage naval}

Avec le peintre et illustrateur Pierre Gatier, les artistes nationaux contribuent aussi, à partir du printemps 1916, à la mise au point de solutions de camouflage en contexte naval, d'abord adoptées par la Royal Navy, la plus exposée aux attaques de la flotte de surface et sous-marine allemande. Mais face à l'intensification de la guerre menée par les U-Boote à partir de janvier 1917, les camoufleurs britanniques Cecil King et Norman Wilkinson dépassent rapidement les recherches françaises dans le domaine de la déstructuration visuelle avec la " Dazzle Painting " ou " peinture tape-à-l'œil ". Également employée dans l'aviation, cette technique consiste à tromper un observateur doté d'un télémètre à coïncidence quant à la distance, à la vitesse, au cap et au type - et donc aux dimensions - des bâtiments, au moyen de zébrures et d'enchevêtrements de lignes droites colorées ou non, multipliant les effets d'ombres et de lumières, déstructurant les volumes et brouillant les contours.

La création avant-gardiste a là-aussi, et peut-être plus largement, intégré ce moyen de camouflage fondé sur l'illusion d'optique dans un contexte au statut artistique ouvertement assumé. C'est le cas par exemple de la photographe et plasticienne new-yorkaise Carrie Schneider avec sa photographie de 2008 au titre sans équivoque de Dazzle Camouflage. Comme l'a souligné le critique Paul Germanos à propos de cette ouvre, le terme "Dazzle " renvoie à une " technique de champ de bataille qui perturbe la perception de l'adversaire grâce à l'utilisation de motifs très contrastés, totalement étrangers à l'objet ainsi traité22 ". Il ne s'agit donc pas pour Carrie Schneider de dissimuler le canoë photographié en situation au milieu d'un lac de Finlande, ainsi qu'en témoigne son choix d'un contexte lumineux accentuant l'effet de contraste, mais bien, comme l'indique l'artiste qui dit avoir cherché à valoriser l'effet de miroitement, de brouiller la lecture de l'objet en situation. Ainsi qu'elle l'explique : "J'aime l'illusion que je peux créer dans une image, comme la façon très simple dont les éléments peuvent être mis ensemble pour faire quelque chose qui est plus que la somme de ses parties ${ }^{23}$."

21. LALANCE, Jean, " Les artistes lorrains camoufleurs ", Le Pays Lorrain et le pays messin, revue régionale mensuelle illustrée, Nancy, quinzième année, $\mathrm{n}^{\circ} 8$, août $1923, \mathrm{p} .373$.

22. Germanos, Paul, "Carrie Schneider @ Monique Meloche », [compte-rendu de l'exposition de C. Schneider How Not To Fall, 17 octobre-6 décembre 2008, Monique Meloche Gallery, Chicago], Chicago Gallery Review, 17 octobre 2008, [http://badatsports.com/2008/ chicago-gallery-review-carrie-schneider-monique-meloche-lora-fosberg-linda-warren-amymayfield-threewalls/]. Consulté le 12 août 2014. Nous traduisons.

23. SCHNEIDER, Carrie, dans "Carrie Schneider \& Salla Tykkä, in conversation ", Helsinki, 13 avril 2008, [http://www.carrieschneider.net/text/CS-ST_conv.pdf]. Consulté le 15 septembre 2014. Nous traduisons. 
En 2008, Jeff Koons s'inspire lui aussi de la Dazzle Painting pour l'animation des surfaces externes du yacht dessiné par Ivana Porfiri pour le collectionneur et industriel Dakis Joannou. L'artiste new-yorkais, promoteur d'un système d'expression kitsch markétisée, évoque bien cette antériorité militaire en définissant la peinture " tape-à-l'œil " comme " une technique de camouflage utilisée durant la Première Guerre mondiale, non pour dissimuler un objet mais pour tromper l'observateur ". Significativement, Koons indique que les formes rythmant les flancs du yacht, conçues dans son studio selon un procédé de modélisation $3 \mathrm{D}$, " dialoguaient avec l'idée de pyramides et une image d'oasis et de mirage ". Dans ce cas encore, le reflet fait potentiellement partie des modalités de lecture de l'œuvre : «Si la mer était calme, alors il y aurait une réflexion, l'effet de dédoublement rendrait le motif encore plus abstrait ${ }^{24}$."

Si le parti décoratif adopté par Koons répond dans le principe à l'objectif militaire de la tromperie visuelle, il sert aussi une ambition en totale rupture, déjà convoquée chez Schneider : celle de la démarcation. Comme l'écrit Augustino Fontevecchia, journaliste du webzine Forbes, les alternances de losanges jaunes, de triangles roses et de polygones bleus ne permettent manifestement pas de " cacher " l'œuvre d'art flottante de Joannou dans un environnement maritime ${ }^{25}$. Conformément au principe même de la Dazzle Painting, la réinterprétation de la peinture géométrique propre au camouflage naval, associée à la technique d'impression tramée intégrant les points dits "Ben-day " employés par Roy Lichtenstein et encore Alain Jacquet, surexpose l'objet-support. Un article non signé du webzine Jetsetmagazine.net daté du 9 janvier 2014 décrit ainsi la réalisation comme une " création démesurée qui transforme la navigation en une expérience unique et ne laisse ou laissera personne indifférent ". Et cela d'autant plus que cette interpellation visuelle est le fait de l'un des créateurs contemporains les plus célèbres, riches et controversés, que l'auteur présente comme " un artiste cultivant le kitsch, très apprécié par des milliardaires nouveau-riches, mais beaucoup moins par la critique ». Le journaliste écrit encore : "Du camouflage donc pour un artiste ultra médiatisé qui en une dérobade médiatique savamment orchestrée nous signifie sa capacité à s'approprier une imagerie collective (celle de la guerre) au service d'un collectionneur "nouvellement" armateur. Une rencontre sulfureuse entre art et économie ${ }^{26}$."

24. Jeff Koons, cité dans Fontevecchia, Augustino, " Dakis Joannou's Mega Yacht "Guilty" By Jeff Koons And Ivana Porfiri, An Act Of Calculated Irreverence ", Forbes.com, 26 novembre 2013, [http://www.forbes.com/sites/afontevecchia/2013/11/26/dakis-joannous-mega-yacht-guilty-by-jeff-koons-and-ivana-porfiri-an-act-of-calculated-irreverence/?ss=giftguide]. Consulté le 16 septembre 2014. Nous traduisons.

25. FONTEVECCHIA, Augistino, "Dakis Joannou's... », op. cit.

26. Anonyme, « Un magnifique Yacht designed by Jeff Koons : GUILTY! », Jetsetmagazine. net, 9 janvier 2014. [http://www.jetsetmagazine.net/jetset,mag/un-magnifique-yacht-designed-by-jeff-koons-guilty.16.2421.html]. Consulté le 3 novembre 2014. 
La spectaculaire proposition de Koons peut être rapprochée d'un projet aux dimensions nettement moins ambitieuses conçu en 2010 par Arian Bantjes. La créatrice canadienne recycle en contexte artistique la technique de la Dazzle Painting en répondant à une commande de décoration d'un dériveur de type Laser. On retrouve, tout au moins dans le discours, la double logique de la désorientation visuelle de l'observateur et de la démarcation de l'œuvre-objet. Citons Arian Bantjes :

" J'ai décidé d'éviter l'évidence consistant à travailler avec des formes organiques s'accordant avec le vent, l'eau, etc, pour travailler délibérément contre ces formes. Je voulais faire quelque chose d'incroyablement identifiable dans l'eau, et qui déguiserait sa vitesse et sa polyvalence. Créer une illusion d'optique, quelque chose qui n'aurait pas l'air d'appartenir du tout à l'eau, tout comme les motifs cubistes du camouflage naval de la Première et de la Seconde Guerre mondiale ${ }^{27}$."

L'artiste, ici, apporte à une production initialement standardisée une originalité formelle dupliquée toutefois à 12 exemplaires, de la même façon que les camoufleurs de la Grande Guerre adaptaient aux réalités du terrain les productions normalisées des ateliers de camouflage : grillage, voilette, toile forte, peinture, filets, guérites blindées, observatoires sur pylône métallique démontables...

Un an après sa découverte du "Dazzle camouflage ", lors d'une visite de l'exposition Architecture en uniforme, projeter et construire pour la Seconde Guerre mondiale présentée au Centre Canadien d'Architecture de Montréal du 13 avril au 18 septembre 2011, c'est une démarche d'adaptation similaire que s'applique à suivre Alexis Judic avec sa série Brassioux (figure 57), du nom de la cité pavillonnaire castelroussine bâtie au début des années 1950 pour les officiers de l'US Air Force sur un modèle de cité-jardin étatsunienne. L'artiste diplômé de l'École des Beaux-Arts du Mans, qui se définit comme un " archéo-anthropologue des "formes perdues" 28 ", explique à propos de l'œuvre Dazzle eagle (figure 58) préfigurant cet ensemble exécuté dans le contexte d'une résidence à Châteauroux :

" Dazzle eagle est une peinture dont le châssis reprend la forme d'une maison pavillonnaire où vient s'appliquer un motif de camouflage "dazzle" (camouflage utilisé sur les bateaux militaires durant la première guerre mondiale). Ici, l'architecture type, conçue selon un modèle et répétée comme une matrice, où toutes les habitations se ressemblent est alors camouflée, dissimulée pour mieux réapparaître ${ }^{29}$."

27. BANTJES, Arian, « Laser Sailboat ", Bantjes.com, 2010, [http://bantjes.com/work/lasersailboat/]. Consulté le 5 novembre 2014.

28. JuDIC, Alexis, "Présentation ", Alexisjudic.com, [http://alexisjudic.com/about/]. Consulté le 6 août 2015.

29. Judic, Alexis, "Communiqué de presse " de l'exposition Alexis Judic, Chateauroux. Collège Marcel Duchamp, 12 janvier-6 février 2013, [http://www.paris-art.com/agenda-culturel-paris/alexis-judic/alexis-judic/14442.html]. Consulté le 6 novembre 2014. 
Le plasticien, qui rapporte avoir « tout de suite été fasciné par ces motifs en relation avec l'histoire de la peinture et aussi par le fait que l'armée ait fait appel à des artistes pour les réaliser ", indique que la déclinaison systémique du principe éprouvé avec Dazzle eagle lui permet de " jouer avec l'illusion d'optique créée par les motifs entrecroisés et redessiner une forme à l'intérieur de la peinture ", et encore de " jouer avec cette idée de dissimuler l'architecture pavillonnaire sérielle derrière un motif de camouflage et en même temps de révéler une architecture " désormais affranchie de la " sujétion matricielle ${ }^{30}$ ".

Dans sa résonnance historique, la Dazzle Painting est étroitement associée à son application navale. Significativement, le canadien Robert Gordon McHarg III y recourt en février 2011 pour transformer en sous-marin une galerie d'art installée dans le métro de Londres, à partir d'une base documentaire textuelle, graphique et photographique. Une démarche de travestissement qui prend le contrepied de celle consistant, pour les marines française et britannique, à maquiller des bâtiments d'attaques en inoffensifs navires civils, dans le cadre de la pratique dite des "Q-Ships " ou " Bateauxpièges ", à la mise au point desquels contribua le docteur Jean-Baptiste Charcot. Après l'évocation, sur le ton humoristique, d'une bataille menée dans le cadre d'une mission souterraine au service des arts, le court texte qui présente cette transformation donne une brève définition de la peinture " tape-à-l'œil " faisant explicitement référence au camouflage naval britannique de la Grande Guerre et citant Norman Wilkinson :

«Durant la Première Guerre mondiale, les Britanniques et les Américains durent faire face à la grave menace que faisaient peser les U-boots allemands sur les convois alliés, dont la destruction atteignait un niveau critique. Toutes les tentatives de camouflage des navires avaient échoué, l'aspect de la mer et du ciel étant en changement constant. Tout système de couleur dissimulant les bateaux dans un contexte particulier les rendaient visibles dans un autre. Un artiste britannique et officier de marine, Norman Wilkinson, promut un nouveau système de camouflage : la Dazzle Painting. Au lieu d'essayer de dissimuler le navire, elle en rompait simplement les lignes, rendant difficile la détermination de son cap par un capitaine de U-Boot ${ }^{31}$."

On l'a vu, la Dazzle Painting n'a pas été employée par les artistes contemporains qu'à partir d'un support navigant, conformément à l'usage premier. On en trouve confirmation dès 1990 avec l'Opel Kadett de Patricia van Lubeck, repeinte à la suite de la visite d'une exposition sur le camouflage maritime de la Première Guerre mondiale au musée maritime Prins Hendrik de Rotterdam. "J'ai essayé, écrit l'artiste hollandaise, de créer un déséquilibre à l'arrière de la voiture, en peignant une sorte de forme oblique autour de la plaque d'immatriculation, les feux arrière et la fenêtre arrière. "

30. JuDic, Alexis, propos recueillis par l'auteur, 25 mars 2016.

31. Anonyme, "Razzle Dazzle, Robert Gordon McHarg III, 10-26 février 2011 ", Subwaygallery.com, [http://www.subwaygallery.com/Razzle\%20Dazzle.html]. Consulté le 2 août 2014. Nous traduisons. 
Patricia van Lubeck, qui a semble-t-il travaillé à ce projet sans anticiper sa réception publique, nous renseigne également sur les réactions suscitées par son œuvre utilisée au quotidien comme véhicule dans la ville hollandaise d'Alkmaar. Elle rapporte sur ce point : " La plupart des gens étaient agréablement surpris. En fait, j'ai même souvent bénéficié d'un droit de passage non règlementaire, donné par des gens qui s'accordaient ainsi quelques secondes de plus pour observer cette chose étrange. " Et encore : "Une autre chose que j'ai remarqué était les places de stationnement libres à côté de ma voiture. Même sur une journée de grande affluence, les espaces libres à côté d'elle sont restés vides le plus longtemps. Peutêtre que les gens avaient peur que quelque fou la fasse sauter et fasse la même chose avec leurs voitures ${ }^{32}$. " Dans une certaine mesure, ce constat apporte une confirmation inattendue aux résultats d'une vaste enquête ouverte par les marines française et anglaise à la fin de la Première guerre, s'attachant notamment à déterminer l'efficacité de la Dazzle Painting. Cette enquête conclut que ce type de camouflage « ne peut pas nuire à un navire et que, même quand il n'arrive pas à tromper sur la route suivie, il doit toujours gêner le sous-marin, le forçant à tenir le périscope au-dessus de la surface de la mer beaucoup plus longtemps que d'habitude ${ }^{33}$ ". Cela peut être capital : quelques secondes ou minutes perdues par un sous-marin dans l'observation de sa cible peuvent faire échouer son attaque et le faire repérer par les avions et les navires d'escorte. Significativement, dans la dernière année du conflit, les commandants de U-Boot étaient soumis à un entraînement spécifique les préparant à l'attaque des bâtiments camouflés.

En février 2014, sur son blog, le photographe et plasticien Romain Sandt lance cet appel aux internautes : "Si vous avez une voiture blanche ou noire et que vous voulez tentez l'expérience n'hésitez pas à me faire signe! " L'artiste, qui présente sur sa page une simulation de camouflage d'une SAAB 9-3, souhaite en effet prolonger effectivement l'expérience de Van Lubeck combinée à celle menée par Sonia Delaunay sur sa Citroën B12 en 1925. Deux sources d'influence qui ont nourri le travail de Romain Sandt sur le modèle vivant (en collaboration avec la maquilleuse Mélissa Gougeon), conduit dans le cadre de la série Dazzle ainsi introduite : "Inspirée par les peintures appliquées sur les Navires pendant la première guerre mondiale appelée DAZZLE PAINTING, cette série est un travail sur la confusion de la lecture du corps dans l'image ${ }^{34}$."

En 2010, la danoise Alexandra Reimann s'inspire également de la Dazzle Painting pour créer une installation à partir d'un artefact utilitaire renvoyant

32. VAN LUBECK, Patricia, "The Art Cars ", Vanlubeck.com, [http://www.vanlubeck.com/ other-projects/]. Consulté le 5 août 2014.

33. Rapport des commandants de sous-marins à l'Amirauté britannique, 1918. Cité dans Compère-Morel, Thomas, Harel, Véronique (dir.), Camouflages, cat. exp., Péronne, Historial de la Grande Guerre, 1997, p. 95-96.

34. SANDT, Romain, « Dazzle painting on Car Saab 9-3", Romainsandt.blogspot.fr, [http:// romainsandt.blogspot.fr/2014/02/dazzle-painting-on-car-saab-9-3.html], 26 février 2014. Consulté le 10 août 2014. 
là-encore à l'environnement quotidien, en l'occurrence une brouette, dont elle contrarie l'identification immédiate. Il en va de même deux ans plus tard pour les objets intégrés aux deux installations du Mexicain Francisco Moreno Las Noticias (robe, affiche publicitaire, chariot à roulettes, planches de bois...) et Dormant Subversion (guéridon, violoncelle, canapé, chaise, lampe...). En mai 2013, dans un entretien accordé à la revue Glasschord, l'artiste assume lui-aussi la référence à la Grande Guerre à propos de l'installation Las Noticias en rappelant l'origine de la Dazzle Painting - dans son exploitation états-unienne - et en citant également Wilkinson :

"En 1917, a été créé le Service américain du camouflage. L'organisation a embauché le peintre britannique Norman Wilkinson afin d'initier l'utilisation de la "peinture d'éblouissement" pour le camouflage naval. Cette peinture a été fortement influencée par le cubisme en ce sens qu'elle consiste en une rupture de contours, $[\ldots]$ de sorte que la forme se confond avec l'espace autour de lui ou avec d'autres forme : des avions ou des tons; contours qui coïncident avec d'autres contours, puis réapparaissent soudainement dans de nouvelles relations : les surfaces qui reculent et avancent par rapport à d'autres surfaces en même temps; parties d'objets décalés loin, déplacés ou modifiés dans le ton jusqu'à ce que la forme disparaisse derrière eux. Ces schémas de peinture ont été créés par des artistes et exécutés par des ouvriers $^{35}$."

Mais surtout, comme Arian Bantjes, Moreno inscrit sa proposition dans la cohérence de la recherche picturale initiée par Picasso avec le Cubisme analytique, menée jusqu'aux limites de la figuration. Significativement, l'artiste explique que c'est en découvrant la Dazzle Painting qu'il a pu véritablement donner sens à la peinture abstraite. L'expérience fait écho à celle vécue par Fernand Léger - candidat malheureux au service français du camouflage en dépit des mises en garde de son ami André Mare ${ }^{36}$-, lequel écrivait depuis l'Argonne en mai 1915 :

"Cette guerre-là, c'est l'orchestration parfaite de tous les moyens de tuer, anciens et modernes. C'est intelligent jusqu'au bout des ongles. [...] C'est linéaire et sec comme un problème de géométrie. Tant d'obus en tant de temps sur une telle surface, tant d'hommes par mètre et à l'heure fixe en ordre. Tout cela se déclenche mécaniquement. C'est l'abstraction pure, plus pure que la Peinture cubiste "soi-même"."

Le peintre, quelques mois plus tard, trouvera à Verdun " des sujets tout à fait inattendus et bien faits pour réjouir [son] âme de cubiste ${ }^{37}$ ".

35. Moreno, Francisco, "Las Noticias ", Glasschord, vol. 2/9, 1 ${ }^{\mathrm{er}}$ mai 2013, [http:// glasschord.com/francisco-moreno/las-noticias/]. Consulté le 8 juin 2014. Nous traduisons.

36. Mare s'est en effet employé à lui démontrer que " le camouflage [était] le plus beau truc possible pour se faire casser la gueule " (A. Mare, lettre à Charlotte Mare, citée dans GrafFIn, Laurence, André Mare. Carnets de guerre 1914-1918, Paris, Herscher/Association André Mare, 1996, p. 58). Nombreux ont été les camoufleurs qui " trouvèrent une mort ou des blessures glorieuses " ([Anonyme], Bulletin de la section d'information du GQG, Nouvelle série no 91, 16 février 1919, p. 4).

37. LÉGER, Fernand, "Une correspondance de guerre à Louis Poughon, 1914-1918. Texte présenté, établi et annoté par Christian Derouet ", Cahiers du musée national d'art 
La recherche d'une "pureté abstraite " a été l'une des intentions des Vorticistes évoqués plus haut par Lakonik, notamment pour parvenir à la formulation de réalités propres à la guerre et à son expérimentation physique et mentale. L'un de ses principaux acteurs de ce mouvement brièvement actif de 1914 à 1915, Percy Wyndham Lewis, assurait ainsi : " Il n'y a pas de réelle raison, ni de place, à faire l'éloge des soldats, sauf par la voie d'un hymne abstrait ${ }^{38}$. " En 2009-2010, l'artiste anglais Carl Fudge crée une série d'œuvres directement inspirée par la production du Vorticiste Edward Wadsworth, camoufleur naval pour la marine britannique à partir de 1917. Après quelques expériences abstraites menées au début de la guerre, Wadsworth ne quittera plus l'expression figurative à partir de 1918, même si ses représentations de bateaux camouflés ou d'installations portuaires frôleront souvent la non-figuration. Dans sa démarche de réappropriation, Carl Fudge atteint la même limite au moyen de l'outil informatique avec Disruption (2009) et encore Aground (2010), dérivés du Dazzle-ships in Drydock at Liverpool peint par Wadsworth en 1919, ou bien franchit cette limite et annule toute possibilité d'identification immédiate des sujets, comme avec Plate Layers 2 de 2010.

En 2011, le lien entre Dazzle Painting et abstraction est également établi par le français Jean-Baptiste Sauvage avec deux projets réalisés à Marseille, prenant pour support une grille de devanture et un container de transport (figure 59). Sur le site internet de l'artiste, le texte qui présente les deux œuvres, dans lequel l'on retrouve la référence pédagogique, et peut-être encore une fois légitimante, au camouflage de la Grande Guerre, inscrit bien les propositions dans la logique picturale non figurative. On peut y lire : " En tension pour ce projet, différents champs de référence, peinture forme/ fonction, déconstruction du volume par le motif, problématique de la représentation dans la filiation des développements de l'abstraction picturale ${ }^{39}$."

Toujours en 2011, cette fois à New York, Charles Mary Kubricht investit aussi le container afin, dit-il, de le rendre " méconnaissable plutôt qu'invisible " en faisant "interagir deux systèmes de perception visuelle, l'un immédiat et empirique, l'autre indirect et technique ". L'artiste, qui inscrit son œuvre dans un environnement architectural intégrant a minima la donnée naturelle, entend " réorganiser notre expérience perceptive en réduisant la tension entre nature et culture ", en assumant la transformation de la « forme industrielle " en " forme esthétique ${ }^{40}$ ». Il rejoint ainsi l'objectif fondamental affiché par Francisco Moreno, lequel explique utiliser la Dazzle

moderne, Paris, Centre Georges Pompidou, 1990, p. 36.

38. PÉPIN, Carl, "Les artistes d'avant-garde au combat. Évolution et redéfinition de la pratique de l'art pendant la grande guerre ", Guerres mondiales et conflits contemporain, 13 janvier 2011, [http://carlpepin.com/tag/vorticisme/]. Consulté le 7 septembre 2014.

39. Sauvage, Jean-Baptiste, "Razzle Dazzle \#2 », 2011, Jb-sauvage.com, [http://www.jbsauvage.com/index.php?an=2011\&projet=118]. Consulté le 26 novembre 2014.

40. KuBRICHT, Charles Mary, dans Tabitha PISENO, « Charles Mary Kubricht's Alive-nesses : A Proposal for Adaptation by Tabitha Piseno ", Bombmagazine.org, 4 janvier 2012, [http:// bombmagazine.org/article/6340/]. Consulté le 6 juin 2014. Nous traduisons. 
Painting de la même manière que le cubiste Picasso dans sa phase analytique, soit pour " trouver une expérience esthétique en marge de ce qui a été réglementé socialement, puisque ce n'est qu'à partir de ce moment que l'artiste avancé peut construire une image de la liberté ${ }^{41}$ ».

La concentration exceptionnelle d'artistes au sein du service du camouflage français de la Grande Guerre explique le climat particulier qui pouvait y régner, transposant en contexte militaire l'atmosphère des ateliers de l'École des Beaux-Arts. Comme l'a rapporté Henri Guingot, les « conversations, les discussions, les échanges de vues y étaient souvent du plus haut intérêt : et le profit tiré par les jeunes du contact permanent d'anciens de si grande valeur, ne pouvait être que considérable. On se plaisait aussi à faire revivre les épisodes joyeux des années d'écoles ${ }^{42}$ ". Cette proximité des camoufleurs - que l'on se gardera bien de généraliser en gardant à l'esprit le témoignage d'André Mare, notamment ${ }^{43}$ - a certainement concouru au dépassement des inévitables tensions causées par les querelles d'hommes, les jalousies d'artistes ou encore les désaccords esthétiques, et ainsi à la mobilisation des camoufleurs au profit de leur mission. Une mobilisation bien évoquée en 1917 dans le dernier couplet de la Chanson des camoufleurs de Jacques Folrey :

"Bref, sachez-le bien, Barbouilleurs de l'arrière,

Chez [les camoufleurs] point de Salon, donc point d'bassesses à faire,

Les querelles d'École n'y font même pas leurs frais

C'est l'union sacrée de tous les peintres français ${ }^{44}$ !"

Citons à la suite l'hommage de Guirand de Scevola rendu en 1950 dans la Revue des Deux Mondes:

"Certes, tous les membres de [la] famille [des camoufleurs] n'appartenaient pas aux mêmes idées politiques et artistiques. Les cubistes y côtoyaient les disciples d'Ingres, et les systèmes sociaux les plus divers y comptaient leurs partisans; mais rien de tout cela ne comptait en regard du but à atteindre, de la trouvaille à réaliser. [...] Tous, depuis le sculpteur célèbre, grand dignitaire de la Légion d'Honneur jusqu'au modeste artisan, rivalisaient d'ingéniosité, de dévouement. Le camouflage, c'était leur chose, ils l'aimaient comme on chérit un être vivant ${ }^{45}$."

Pierre Lorain, qui avec son père et certains de ses proches, a côtoyé quelques uns des " rescapés de l'équipe qui avait insufflé vie et âme à la

41. Moreno, Francisco, cité dans Magdaleno, Jonathan, "Abstracting the Fleet: Dazzle Camouflage's Influence on Contemporary Art and Design ", Thecreatorsproject.vice.com, 3 juin 2013, [http://thecreatorsproject.vice.com/blog/abstracting-the-fleet-dazzle-camouflage]. Consulté le 7 juin 2014. Nous traduisons.

42. Guingot Henri, "Les artistes et la guerre... ", op. cit., p. 22.

43. Voir notamment A. Mare, lettre à Charlotte Mare, citée dans GrAFFin, Laurence, André Mare..., op. cit., p. 72.

44. FolREY, "La chanson des camoufleurs ", La Bä̈onnette, Paris, nº 112, août 1917, p. 538 .

45. GuIRAND DE ScÉvola, Lucien Victor, Souvenirs du camouflage..., op. cit., p. 724. 
section ", a significativement parlé d'une " confrérie des camoufleurs ". Il a insisté par ailleurs sur l'abnégation de ces artistes à la "personnalité souvent très forte " en évoquant leur " foi " et la valeur d'un travail " dans lequel ils mettaient le meilleur d'eux-mêmes ${ }^{46}$ ", avec pour premier objectif d'apporter des réponses normalisées ou expérimentales aux demandes de camouflage en augmentation continue ${ }^{47}$. Dans son témoignage de 1934, Henri Guingot a rappelé sur cette question que chaque camoufleur pouvait apporter " sa contribution d'idées nouvelles au fur et à mesure qu'elles [germaient] dans les esprits, suscitées par les hasards de la guerre et la tournure des opérations ${ }^{48}$ ".

La volonté d'implication plus ou moins unificatrice des artistes camoufleurs, renforcée chez beaucoup par la certitude de leur utilité ${ }^{49}$ et une conviction patriotique doit-elle aussi être reliée à un sentiment de nature artistique? Le camouflage comme expérience artistique ou tout au moins esthétique : la question s'est posée plus ou moins clairement aux artistes camoufleurs dans le contexte même de la Grande Guerre et dans les années qui ont suivi. À plus forte raison dans le temps du conflit où la pratique créatrice, généralement contrariée voire empêchée, est souvent ressentie comme un " moyen de ne pas sombrer, de tenir, un moyen d'oublier et de se tenir face à la destruction comme personne vivante, encore ${ }^{50}$ ". Ainsi, Roger de La Fresnaye, qui fait une brève visite à l'atelier de camouflage de Noyon au cours de l'année 1917, interrompt sans doute complètement son activité de septembre 1914 à l'été 1917. Le 5 février 1918, il écrit à André Mare : « Je n'ai plus retrouvé depuis un mois, ni le temps, ni les commodités de faire quoi que ce soit comme peinture ou dessin - Et je m'enfonce dans une vie bien sotte ${ }^{51}$."

Certes, l'exercice du camouflage a pu être une source d'inspiration iconographique venant contourner en partie cette observation faite par le

46. LORAIN Pierre, «L'arme du camouflage ", Gazette des armes, Paris, no 58, mars 1978, p. 16.

47. L'augmentation constante du nombre des interventions et de l'effectif des camoufleurs (3000 artistes, artisans ou chimistes dispersés sur le front en 1918, secondés par 80000 civils employés dans les ateliers produisant des structures de camouflage standardisés), prouve que leur travail est très tôt jugé efficace. Comme l'a rappelé le commandant Lalance, le camouflage suscite dès 1915 un "véritable engouement : on camouflait tout; on camouflait inutilement; on camouflait même beaucoup trop " (J. LALANCE 1923, op. cit., p. 367).

48. Guingot, Henri, "Les artistes et la guerre... ", op. cit., p. 18.

49. Le bulletin de la section d'information du GQG du 19 février 1919 nous apprend par exemple que « 4 p. 100 seulement des batteries [...] camouflées [par filet et grillage] ont été repérées par l'ennemi, tandis que pour les autres la proportion s'élève à 50 p. 100 " ([Anonyme], Bulletin..., op. cit. note 10, p. 3).

50. Tison, Stéphane, « Roger de la Fresnaye : un artiste dans la Grande Guerre », dans LUCBERT, Françoise (dir.), Roger de la Fresnaye (1885-1925). Cubisme et tradition, Rennes, PUR, 2016. Actes du colloque international tenu à l'université du Maine les 20 et 21 janvier 2006. Voir aussi MaINGON, Claire, « Hypermobilité : L'évolution des pratiques artistiques du temps de la Grande Guerre ", Pays Riverains de la Baltique, Sylvains-les-Moulins, n 4, 2007, p. 129-140.

51. Roger de la Fresnaye, lettre à André Mare, 5 février 1918, IMEC. Fonds André Mare, lettre $n^{\circ} 24$. Cité dans Tison, Stéphane, "Roger de la Fresnaye... ", op. cit. 
critique Camille Mauclair en 1918 : " [La guerre] enlève systématiquement à l'artiste toutes les raisons d'intervenir. [...] le malheureux peintre [...], possesseur d'un art qui exprime le visible, doit capituler devant un spectacle où la première condition est de tout cacher au regard ${ }^{52}$. "Ou encore ce constat bien connu formé par Fernand Léger dans une conférence de 1937 : " La guerre fut grise et camouflée : une lumière, une couleur, un ton étaient interdits sous peine de mort. Quatre années sans couleur ${ }^{53}$." Nous avons évoqué le cas d'Edward Wadsworth et de ses représentations de navires camouflés. Nous pourrions encore songer à l'Anglais Leon Underwood montrant l'installation d'un arbre-observatoire dans sa toile de 1919 Erecting a Camouflage Tree, aux Français André Devambez représentant une opération dite de "barbouillage " d'une pièce d'artillerie dans son tableau titré Camouflage (peint entre 1915-1918) et Alfred-Marie Le Petit livrant une vue de l'atelier de préparation de toiles colorées installé aux Buttes-Chaumont, avec une gouache sans titre de 1918.

Au-delà, s'il a sans doute été réduit à une nécessité technique par une majorité d'artistes travaillant hors du champ avant-gardiste et en particulier cubiste, le camouflage a pu être considéré comme un travail de création artistique autonome. Henri Bouchard, également auteur de leurres anthropomorphes, comparait ainsi ses arbres-observatoires à des sculptures. Quant au peintre allemand Franz Marc, il avouait " éprouver d'étranges sensations en peignant des taches sur des toiles militaires ${ }^{54}$ ", un travail qu'il rapprochait des peintures abstraites de Vassily Kandinsky.

Évoquées ici à travers une base d'œuvres et de pratiques réduite, les appropriations artistiques - revendiquées comme telles - de solutions de camouflage de la Première Guerre mondiale ne sont bien sûr pas les seules résonnances ou mémoires du conflit dans l'art actuel. Parmi un abondant corpus en constant enrichissement, pensons au recyclage de l'objet issu de la guerre ou évocateur du conflit, par exemple sur le mode quantitatif, en considérant l'Hommage aux poilus bricoleurs de Jean-Jacques Lebel (2012), collection d'objets représentatifs de l'art ou artisanat de tranchées installée notamment au Centre Pompidou Metz du 26 mai au 24 septembre 2012 pour l'exposition 1917. Songeons encore à Jan Fabre et son accumulation ordonnée de croix et de cocardes mortuaires intégrée à l'exposition collective Fantômes et cauchemars, organisée par l'Historial de la Grande Guerre du 13 mai au 21 août 2011. Une installation qui interroge le rapport entre l'esthétisation mémorielle et la mémoire traumatique de la guerre, a priori étrangère à la pratique de ce que l'on pourrait appeler le néocamouflage artistique.

52. MAUCLAIR, Camille, "L'œuvre de guerre de Steinlein ", L'art et les artistes, Paris, mars 1918, p. 7-8.

53. LÉGER Fernand, "La couleur dans le monde ", conférence de 1937, cité dans PrAT, Jean-Louis (dir.), Fernand Léger. Rétrospective, cat. exp., Saint-Paul-de-Vence, Fondation Maeght, 2 juillet-octobre 1988, p. 52.

54. MARC, Franz, Lettres du front, Paris, Fourbis, coll. "SH », 1996, p. 165. Traduit de l'allemand par Laurent Bonzon. 


\section{RÉSUMÉ}

Le camouflage matériel appliqué durant la Grande Guerre est apparu comme une nécessité immédiate s'appliquant en contexte terrestre et naval dans la perspective défensive et offensive. Fait nouveau dans l'histoire militaire, sa théorisation comme son application ont principalement été confiées à des artistes et artisans d'art dont les compétences spécifiques ont été sollicitées notamment pour la dissimulation d'objectifs soit par fusion homotypique avec les milieux d'implantation, soit par déstructuration des formes révélatrices au moyen de procédés graphiques et chromatiques.

Les démarches plasticiennes contemporaines ont pu croiser ce champ du camouflage sans que leurs initiateurs (A. Jacquet, A. Warhol, E. Rutten, K. Meyffret, S. Lang, R. Lichtenstein, M. Aubry, T. Hirschorn) aient toujours une conscience pleine et documentée de ce qu'ils devaient aux camoufleurs de la Grande Guerre et à leur contribution multiforme. D'autres ont clairement corrélé leurs pratiques à cette dernière - filiation à valeur de légitimation peut-être parfois délibérément recherchée - réinvestie et interprétée dans l'optique terrestre de la dissimulation ou bien au contraire dans celle de la Dazzle Painting ou " peinture tape-à-l'œil " navale (Lakonik, J.-C. Le Gouic, J. Koons, A. Bantjes, A. Judic, R. G. McHarg III, P. Van Lubeck, A. Reimann, F. Moreno, C. Fudge, J.-B. Sauvage, C. M. Kubricht). Tous ont assumé la dimension artistique voire esthétique du camouflage, niée, pressentie ou pleinement identifiée par ses expérimentateurs initiaux parmi lesquels $\mathrm{H}$. Bouchard, F. Marc et $E$. Wadsworth. Une dimension très souvent autonome, faisant du " néo-camouflage " une expression étrangère à l'interrogation de la mémoire traumatique de la guerre.

\section{ABSTRACT}

Material camouflage applied during the Great War emerged as an immediate necessity in both land and naval contexts, in offensive and defensive situations. Its theory as well as its application were new to military history and it was mainly entrusted to artists and craftsmen whose specific skills were requested in particular with regard to the concealment of goals either by merging with homotypical implementation environments, or by the disintegration of revealing forms using graphical and chromatic processes.

Contemporary visual artists approaches integrated such camouflage techniques without their initiators (figures such as A. Jacquet, A. Warhol, E. Rutten, K. Meyffret, $S$. Lang, R. Lichtenstein, M. Aubry, T. Hirschorn) being fully aware of what they owed to the camouflage artists of the Great War and their multifaceted contribution. Other practices were more conscious of their debt - a process of filiation and legitimisation that may sometimes have been deliberately sought - reinvested and interpreted in the vision of terrestrial war concealment or rather in that of naval Dazzle Painting (Lakonik, J.-C. Le Gouic, J. Koons, A. Bantjes, A. Judic, R. G. McHarg III, P. Van Lubeck, A. Reimann, F. Moreno, C. Fudge, J.-B. Sauvage, C. M. Kubricht). All these artists have accepted the artistic and aesthetic dimensions of camouflage that had been either denied or fully recognised by its original experimenters such as H. Bouchard, F. Marc and E. Wadsworth. This "neo-camouflage" is often an independent artistic expression that owes little to the traumatic memory of the war. 


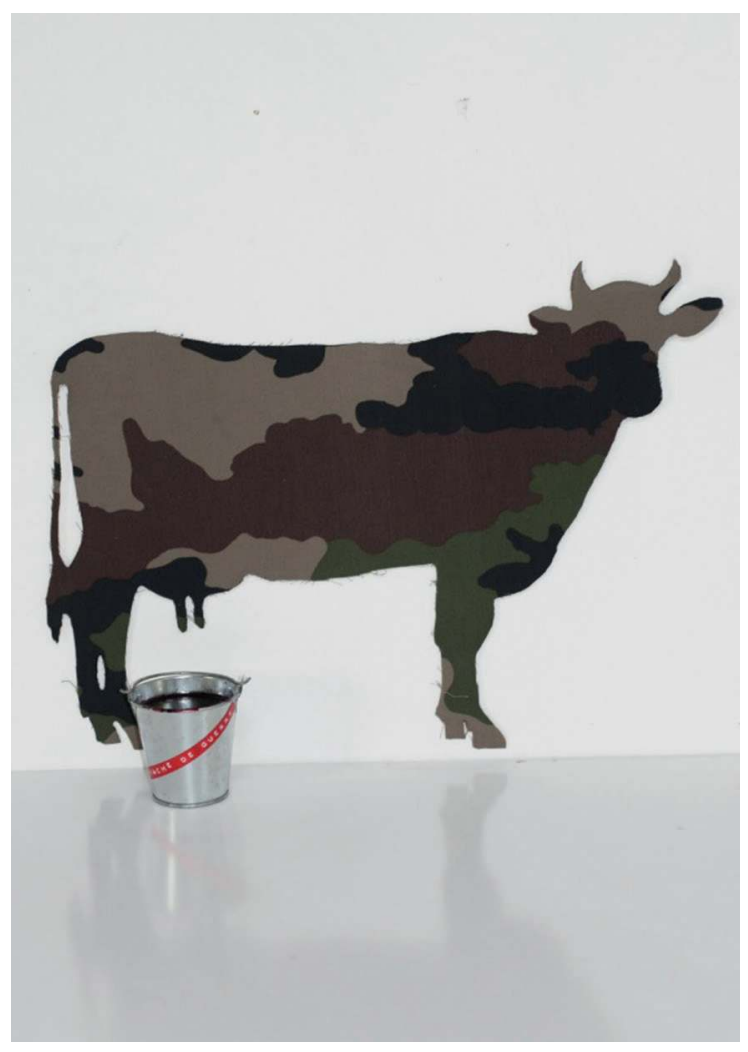

Figure 55 - Louise

Pressager (née en 1985), Vache de guerre, exposition Voyage en Camouflistan, Malzéville, 2011.

Figure 56 - JeanClaude Le Gouic (1944), Camouflages fluorescents, grotte des Perles, Barjols (Var), mars 2011.

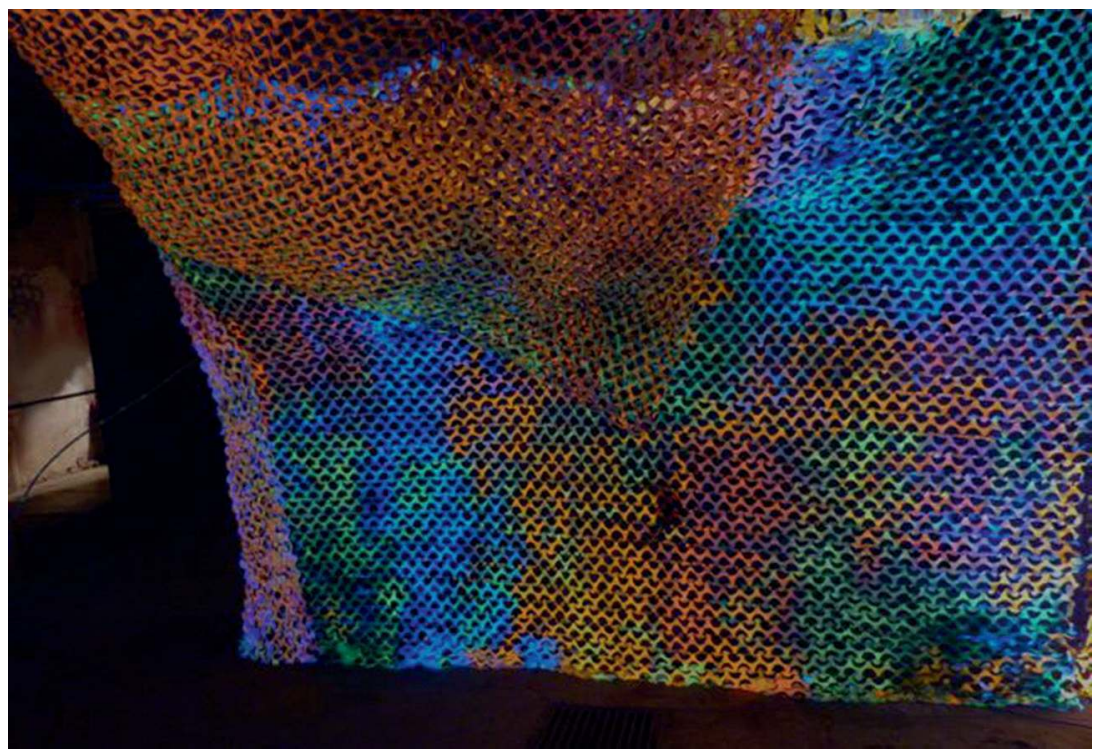




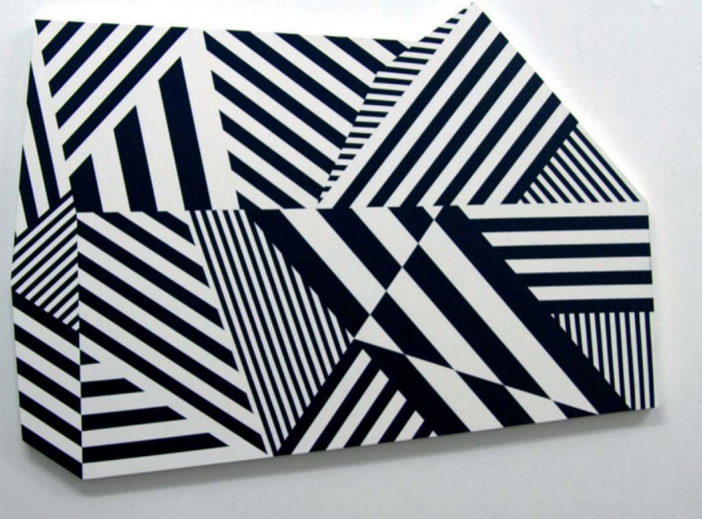

Figure 57 - Alexis Judic (1983), Elise (pièce de la série Brassioux), 2012, peinture sur bois, 120 x $80 \mathrm{~cm}$, coll. part.

Figure 58 - Alexis Judic (1983), Dazzle eagle, 2012, peinture sur bois, $120 \times 80 \mathrm{~cm}$, coll. part.

Figure 59 - JeanBaptiste Sauvage (1977), Razzle Dazzle \#2, 2011, Port SaintLouis, France, Mai 2011.
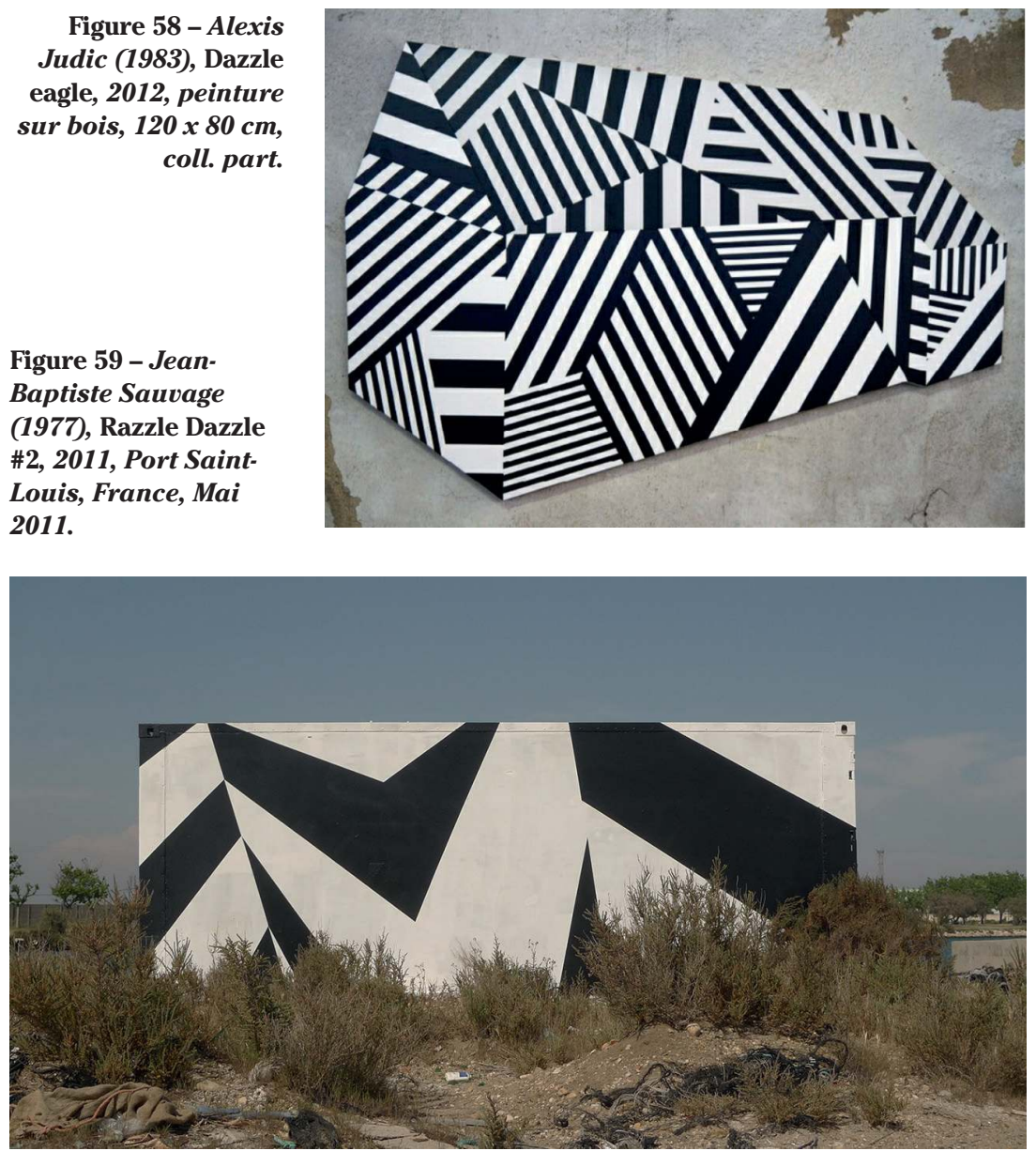